\title{
Structural and Biophysical Properties of Supercharged and Circularized Nanodiscs
}

Nicolai Tidemand Johansen ${ }^{\dagger}$, Alessandra Luchini ${ }^{\dagger}$, Frederik Grønbæk Tidemand ${ }^{\dagger}$, Simone Orioli ${ }^{\dagger, \dagger}$, Anne Martel ${ }^{\S}$, Lionel Porcar ${ }^{\S}$, Lise Arleth ${ }^{\dagger}$, Martin Cramer Pedersen ${ }^{\dagger}{ }^{*}$

'Structural Biophysics Group, Niels Bohr Institute, University of Copenhagen, 2100 Copenhagen E, Denmark

${ }^{\sharp}$ Structural Biology and NMR Laboratory and Linderstrøm-Lang Centre for Protein

Science, Department of Biology, University of Copenhagen, 2200 Copenhagen N, Denmark

\$Institut Laue Langevin, 38000 Grenoble, France

*Corresponding author 


\section{Supporting Information}

Protein sequences

Figure S1 - SAXS data

Table S 1 - X-ray scattering lengths

Table S 2 - Refined parameters from SAXS modelling

Table S 3 - Thermal expansion coefficients for DMPC in nanodiscs

Figure S 2 - SEC-SAXS on supercharged circularized nanodiscs

Figure S 3 - Successive DSC scans

Table S 4 - Lipid exchange rates at different concentrations

Figure S 4 - TR-SANS temperature series

Table S 5 - Thermodynamic parameters from lipid exchange 
Protein sequences

$>$ His-1sMSP1D $1 \Delta \mathrm{H} 5$

$\begin{array}{rrrrrr}10 & 20 & 30 & 40 & 50 & 60 \\ \text { MGHHHHHHHD } & \text { YDIPTTENLY } & \text { FQGSSSSFSK } & \text { LREELGPVSE } & \text { EFWDDLEKES } & \text { EGLREEMSKD } \\ 70 & 80 & 90 & 100 & 110 & 120 \\ \text { LEEVKAKVEP } & \text { YLDDFEKKWE } & \text { EEMELYREKV } & \text { EPLGEEMRDR } & \text { ARAHVDALRS } & \text { HLAPYSDELR } \\ 130 & 140 & 150 & 160 & 170 & 180 \\ \text { ERLAARLEAL } & \text { KEDGGARLAE } & \text { YHAKASEHLS } & \text { SLSEKAKPAL } & \text { EDLREGLLPV } & \text { LESFKVSFLS } \\ 190 & 200 & 210 & & & \\ \text { ALEEYSKKLD } & \text { SEGGRGGSLP } & \text { ETGGASHHH } & \text { HH } & & \end{array}$

ALEEYSKKLD SEGGRGGSLP ETGGASHHHH HH

\begin{tabular}{rrrrrr} 
>HIS-lSMSP1D1 & \multicolumn{2}{c}{} & \\
10 & 20 & 30 & 40 & 50 & 60 \\
MGHHHHHHHD & YDIPTTENLY & FOGSSFSKLR & EELGPVSEEF & WDDLEKESEG & LREEMSKDLE \\
70 & 80 & 90 & 100 & 110 & 120 \\
EVKAKVEPYL & DDFEKKWEEE & MELYREKVEP & LRAELEEGAR & EKLHELEEKL & SPLGEEMRDR \\
130 & 140 & 150 & 160 & 170 & 180 \\
ARAHVDALRS & HLAPYSDELR & ERLAARLEAL & KEDGGARLAE & YHAKASEHLS & SLSEKAKPAL \\
190 & 200 & 210 & 220 & 230 &
\end{tabular}

EDLREGLLPV LESFKVSFLS ALEEYSKKLD SEGGRGGSLP ETGGASHHHH нH

>His-1sMSP1E3D1

$\begin{array}{rrrrrr}10 & 20 & 30 & 40 & 50 & 60 \\ \text { MGHHHHHHH } & \text { YDIPTTENLY } & \text { FQGSSFSKLR } & \text { EELGPVSEEF } & \text { WDDLEKESEG } & \text { LREEMSKDLE } \\ 70 & 80 & 90 & 100 & 110 & 120 \\ \text { EVKAKVEPYL } & \text { DDFEKKWEEE } & \text { MELYREKVEP } & \text { LRAELEEGAR } & \text { EKLHELEEKL } & \text { SPLGEEMRDR } \\ 130 & 140 & 150 & 160 & 170 & 180 \\ \text { ARAHVDALRS } & \text { HLAPYLDDFE } & \text { KKWEEEMELY } & \text { REKVEPLRAE } & \text { LEEGAREKLH } & \text { ELEEKLSPLG } \\ 190 & 200 & 210 & 220 & 230 & 240 \\ \text { EEMRDRARAH } & \text { VDALRSHLAP } & \text { YSDELRERLA } & \text { ARLEALKEDG } & \text { GARLAEYHAK } & \text { ASEHLSSLSE } \\ 250 & 260 & 270 & 280 & 290 & \\ \text { KAKPALEDLR } & \text { EGLLPVLESF } & \text { KVSFLSALEE } & \text { YSKKLDSEGG } & \text { RGGSLPETGG } & \text { ASHHHHHH }\end{array}$

>MSP1D1

$\begin{array}{rrrrrr}10 & 20 & 30 & 40 & 50 & 60\end{array}$

GSTFSKLREQ LGPVTQEFWD NLEKETEGLR QEMSKDLEEV KAKVQPYLDD FQKKWQEEME

$\begin{array}{rrrrrr}70 & 80 & 90 & 100 & 110 & 120\end{array}$

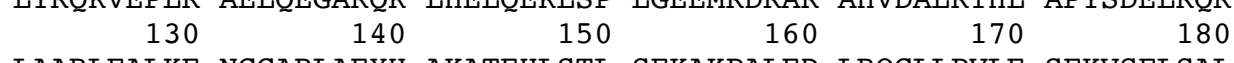

LAARLEALKE NGGARLAEYH AKATEHLSTL SEKAKPALED LRQGLLPVLE SFKVSFLSAL 190

EEYTKKLNTQ

Overview of supercharged MSPs used in this study: The total sequences were those encoded for in the plasmids. Cleaving with TEV protease removes the $\mathrm{N}$-terminal 22 residues, leaving behind what we term linear-solubility enhanced (Is) MSPs (red text). Upon circularization with sortase, the Nterminal $\mathrm{G}$ is connected to the T of the C-terminal LPETG motif. Thus, the circularized- solubility enhanced (cs) MSPs comprise the parts marked in grey and with a covalent bond between the $G$ and T. The sequence for MSP1D1 with the N-terminal His-tag cleaved off is given for comparison. 


\section{Figure S1 - SAXS data}
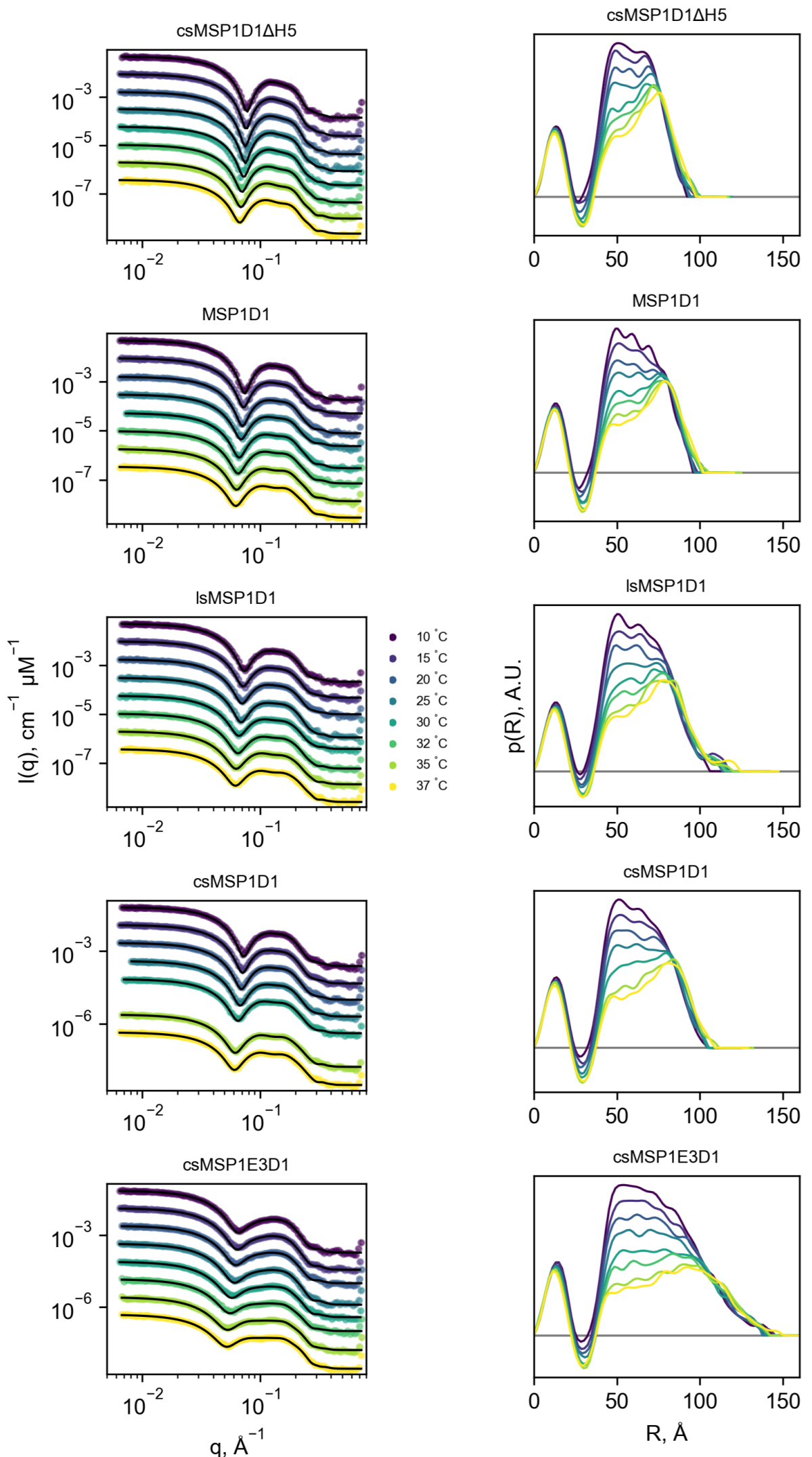

Figure S 1 All collected SAXS data with nanodisc model fits (solid lines) and $p(r)$-distributions. 
Table S 1 - X-ray scattering lengths

\begin{tabular}{|l|l|c|}
\hline Component & Chemical composition & X-ray scattering length, cm \\
\hline DMPC Headgroup & $\mathrm{C}_{10} \mathrm{H}_{18} \mathrm{NO}_{8} \mathrm{P}$ & $4.62 \cdot 10^{-11}$ \\
\hline DMPC Tails & $\mathrm{C}_{24} \mathrm{H}_{48}$ & $5.41 \cdot 10^{-11}$ \\
\hline DMPC Methyl groups & $\mathrm{C}_{2} \mathrm{H}_{6}$ & $5.08 \cdot 10^{-12}$ \\
\hline cSMSP1D1dH5 & $\mathrm{C}_{895} \mathrm{H}_{1416} \mathrm{~N}_{244} \mathrm{O}_{299} \mathrm{~S}_{3}$ & $3.08 \cdot 10^{-9}$ \\
\hline MSP1D1 & $\mathrm{C}_{971} \mathrm{H}_{1561} \mathrm{~N}_{277} \mathrm{O}_{302} \mathrm{~S}_{3}$ & $3.32 \cdot 10^{-9}$ \\
\hline IsMSP1D1 & $\mathrm{C}_{1046} \mathrm{H}_{1648} \mathrm{~N}_{296} \mathrm{O}_{343} \mathrm{~S}_{3}$ & $3.60 \cdot 10^{-9}$ \\
\hline cSMSP1D1 & $\mathrm{C}_{1000} \mathrm{H}_{1590} \mathrm{~N}_{274} \mathrm{O}_{332} \mathrm{~S}_{3}$ & $3.44 \cdot 10^{-9}$ \\
\hline cSMSP1E3D1 & $\mathrm{C}_{1347} \mathrm{H}_{2137} \mathrm{~N}_{373} \mathrm{O}_{442} \mathrm{~S}_{5}$ & $4.63 \cdot 10^{-9}$ \\
\hline Solvent & $\mathrm{H}_{2} \mathrm{O}$ and $\mathrm{D}_{2} \mathrm{O}$ & $2.82 \cdot 10^{-12}$ \\
\hline
\end{tabular}

Table S 1 The chemical compositions and scattering lengths of the components in our SAXS models for nanodiscs. 
Table S 2 - Refined parameters from SAXS modelling

\begin{tabular}{|c|c|c|c|c|c|c|}
\hline Temperature & Parameter & CsdH5 & MSP1D1 & Is1D1 & cs1D1 & csE3 \\
\hline \multirow{3}{*}{ Global } & $N$ & $132 \pm 9.1$ & $151 \pm 9.8$ & $156 \pm 10.8$ & $161 \pm 8.7$ & $245 \pm 14.5$ \\
\hline & $\varepsilon$ & $1.15 \pm 0.29$ & $1.21 \pm 0.20$ & $1.36 \pm 0.19$ & $1.29 \pm 0.15$ & $1.58 \pm 0.19$ \\
\hline & $R, \AA^{2}$ & $4.21 \pm 0.30$ & $3.66 \pm 0.30$ & $4.03 \pm 0.31$ & $3.94 \pm 0.24$ & $4.03 \pm 0.28$ \\
\hline \multirow{4}{*}{$10^{\circ} \mathrm{C}$} & $A, \AA^{2}$ & $55.6 \pm 3.3$ & $55.3 \pm 3.0$ & $55.7 \pm 3.5$ & $55.6 \pm 2.4$ & $55.0 \pm 2.6$ \\
\hline & $V_{D M P C} \AA^{3}$ & $1067 \pm 19$ & $1067 \pm 16$ & $1068 \pm 16$ & $1069 \pm 13$ & $1067 \pm 12$ \\
\hline & $V_{M S P} \AA^{3}$ & $25210 \pm 1468$ & $27352 \pm 1539$ & $29245 \pm 1654$ & $27960 \pm 1245$ & $37210 \pm 1866$ \\
\hline & $b, 10^{-4} \mathrm{~cm}^{-1}$ & $1.40 \pm 1.3$ & $1.78 \pm 1.2$ & $2.09 \pm 1.2$ & $2.37 \pm 1.1$ & $1.83 \pm 1.2$ \\
\hline \multirow{4}{*}{$15^{\circ} \mathrm{C}$} & $A, \AA^{2}$ & $56.3 \pm 3.4$ & $56.0 \pm 3.0$ & $56.8 \pm 3.6$ & $56.6 \pm 2.4$ & $55.7 \pm 2.6$ \\
\hline & $V_{D M P C} \AA^{3}$ & $1073 \pm 19$ & $1076 \pm 16$ & $1078 \pm 16$ & $1077 \pm 12$ & $1074 \pm 12$ \\
\hline & $V_{M S P} \AA^{3}$ & $25210 \pm 1468$ & $27105 \pm 1484$ & $28861 \pm 1566$ & $27819 \pm 1189$ & $37248 \pm 1866$ \\
\hline & $b, 10^{-4} \mathrm{~cm}^{-1}$ & $1.22 \pm 1.3$ & $2.53 \pm 1.2$ & $2.33 \pm 1.2$ & $2.29 \pm 1.1$ & $1.75 \pm 1.2$ \\
\hline \multirow{4}{*}{$20^{\circ} \mathrm{C}$} & $A, \AA^{2}$ & $57.1 \pm 3.5$ & $57.1 \pm 3.1$ & $57.4 \pm 3.7$ & $57.4 \pm 2.5$ & $56.7 \pm 2.7$ \\
\hline & $V_{D M P C}, \AA^{3}$ & $1079 \pm 17$ & $1082 \pm 16$ & $1085 \pm 16$ & $1085 \pm 12$ & $1082 \pm 12$ \\
\hline & $V_{M S P} \AA^{3}$ & $25235 \pm 1417$ & $27270 \pm 1457$ & $28950 \pm 1566$ & $27762 \pm 1160$ & $36867 \pm 1752$ \\
\hline & $b, 10^{-4} \mathrm{~cm}^{-1}$ & $1.09 \pm 1.4$ & $1.97 \pm 1.2$ & $2.44 \pm 1.2$ & $2.46 \pm 1.1$ & $2.40 \pm 1.2$ \\
\hline \multirow{4}{*}{$25^{\circ} \mathrm{C}$} & $A, \AA^{2}$ & $58.1 \pm 3.6$ & $57.9 \pm 3.3$ & $59.0 \pm 3.8$ & $58.4 \pm 2.7$ & $57.9 \pm 2.9$ \\
\hline & $V_{D M P C} \AA^{3}$ & $1090 \pm 17$ & $1093 \pm 15$ & $1090 \pm 16$ & $1092 \pm 13$ & $1089 \pm 12$ \\
\hline & $V_{M S P} \AA^{3}$ & $24932 \pm 1367$ & $26802 \pm 1402$ & $29186 \pm 1536$ & $27677 \pm 1189$ & $37058 \pm 1752$ \\
\hline & $b, 10^{-4} \mathrm{~cm}^{-1}$ & $1.43 \pm 1.3$ & $2.99 \pm 1.2$ & $1.39 \pm 1.3$ & $2.52 \pm 1.2$ & $1.52 \pm 1.3$ \\
\hline \multirow{4}{*}{$30^{\circ} \mathrm{C}$} & $A, \AA^{2}$ & $59.8 \pm 3.8$ & $59.8 \pm 3.4$ & $60.7 \pm 4.2$ & $60.3 \pm 2.7$ & $59.4 \pm 3.1$ \\
\hline & $v_{D M P C} \AA^{3}$ & $1102 \pm 16$ & $1104 \pm 15$ & $1104 \pm 16$ & $1106 \pm 11$ & $1102 \pm 12$ \\
\hline & $V_{M S P} \AA^{3}$ & $24679 \pm 1291$ & $26610 \pm 1319$ & $28536 \pm 1477$ & $27224 \pm 1047$ & $36372 \pm 1676$ \\
\hline & $b, 10^{-4} \mathrm{~cm}^{-1}$ & $1.39 \pm 1.3$ & $1.88 \pm 1.2$ & $2.34 \pm 1.3$ & $2.60 \pm 1.1$ & $2.30 \pm 1.2$ \\
\hline \multirow{4}{*}{$32{ }^{\circ} \mathrm{C}$} & $A, \AA^{2}$ & $61.0 \pm 3.9$ & $60.7 \pm 3.5$ & $61.9 \pm 4.2$ & - & $61.2 \pm 3.9$ \\
\hline & $V_{D M P C} \AA^{3}$ & $1109 \pm 16$ & $1110 \pm 14$ & $1110 \pm 15$ & - & $1110 \pm 13$ \\
\hline & $V_{M S P} \AA^{3}$ & $24476 \pm 1240$ & $26527 \pm 1292$ & $28507 \pm 1388$ & - & $35953 \pm 1828$ \\
\hline & $b, 10^{-4} \mathrm{~cm}^{-1}$ & $1.50 \pm 1.3$ & $2.27 \pm 1.2$ & $1.84 \pm 1.2$ & - & $3.02 \pm 1.4$ \\
\hline \multirow{4}{*}{$35^{\circ} \mathrm{C}$} & $A, \AA^{2}$ & $61.8 \pm 3.9$ & $62.0 \pm 3.6$ & $63.3 \pm 4.3$ & $62.7 \pm 2.9$ & $63.3 \pm 3.5$ \\
\hline & $V_{D M P C} \AA^{3}$ & $1115 \pm 16$ & $1118 \pm 14$ & $1121 \pm 15$ & $1121 \pm 11$ & $1119 \pm 11$ \\
\hline & $v_{M S P} \AA^{3}$ & $24274 \pm 1190$ & $26225 \pm 1210$ & $28034 \pm 1300$ & $26658 \pm 962$ & $35611 \pm 1523$ \\
\hline & $b, 10^{-4} \mathrm{~cm}^{-1}$ & $1.79 \pm 1.2$ & $2.17 \pm 1.2$ & $2.10 \pm 1.2$ & $2.70 \pm 1.1$ & $2.43 \pm 1.2$ \\
\hline \multirow{4}{*}{$37^{\circ} \mathrm{C}$} & $A, \AA^{2}$ & $63.2 \pm 4.1$ & $63.1 \pm 3.7$ & $64.7 \pm 4.5$ & $64.2 \pm 3.0$ & $64.6 \pm 3.7$ \\
\hline & $V_{D M P C}, \AA^{3}$ & $1119 \pm 15$ & $1123 \pm 14$ & $1123 \pm 14$ & $1124 \pm 11$ & $1123 \pm 11$ \\
\hline & $v_{M S P} \AA^{3}$ & $24274 \pm 1164$ & $26088 \pm 1182$ & $28123 \pm 1300$ & $26772 \pm 962$ & $35725 \pm 1523$ \\
\hline & $b, 10^{-4} \mathrm{~cm}^{-1}$ & $1.46 \pm 1.3$ & $2.33 \pm 1.2$ & $1.99 \pm 1.2$ & $2.44 \pm 1.1$ & $2.02 \pm 1.2$ \\
\hline
\end{tabular}

Table S 2 Structural parameters refined from our SAXS data using the outlined modeling scheme 
Table S 3 - Thermal expansion coefficients for DMPC in nanodiscs

\begin{tabular}{|c|c|c|c|c|}
\hline MSP-DMPC & $\begin{array}{c}\alpha_{V, \text { DMPC }}\left(\cdot 10^{-3}\right) \\
K^{-1}\end{array}$ & $\begin{array}{c}\alpha_{A, \text { DMPC }}\left(\cdot 10^{-3}\right) \\
K^{-1}\end{array}$ & $\begin{array}{c}\alpha_{C, \text { DMPC }}\left(\cdot 10^{-3}\right) \\
K^{-1}\end{array}$ & $\begin{array}{c}\alpha_{C, \text { DMPC }} \times C\left(\cdot 10^{-1}\right) \\
\AA K^{-1}\end{array}$ \\
\hline $\operatorname{csMSP} 1 \mathrm{D} 1 \Delta \mathrm{H} 5$ & $1.2 \pm 0.1$ & $2.6 \pm 0.3$ & $1.3 \pm 0.1$ & $2.9 \pm 0.1$ \\
\hline MSP1D1 & $1.5 \pm 0.2$ & $3.3 \pm 0.4$ & $1.6 \pm 0.2$ & $3.7 \pm 0.5$ \\
\hline IsMSP1D1 & $1.4 \pm 0.1$ & $3.1 \pm 0.7$ & $1.5 \pm 0.3$ & $3.6 \pm 0.6$ \\
\hline csMSP1D1 & $1.4 \pm 0.0$ & $3.2 \pm 0.3$ & $1.6 \pm 0.1$ & $3.9 \pm 0.3$ \\
\hline csMSP1E3D1 & $1.4 \pm 0.1$ & $3.1 \pm 0.2$ & $1.5 \pm 0.2$ & $4.6 \pm 0.5$ \\
\hline
\end{tabular}

Table S 3 Thermal expansion coefficients derived from SAXS modeling. Volume thermal expansion coefficient: $\alpha_{V}$, DMPC, lateral thermal expansion coefficient: $\alpha_{A}$, DMPC, linear thermal expansion coefficient of bilayer circumference: $\alpha_{c, \text { DMPC, }} \mathrm{C}$ : circumference of bilayer. 
Figure S 2 - SEC-SAXS on supercharged circularized nanodiscS
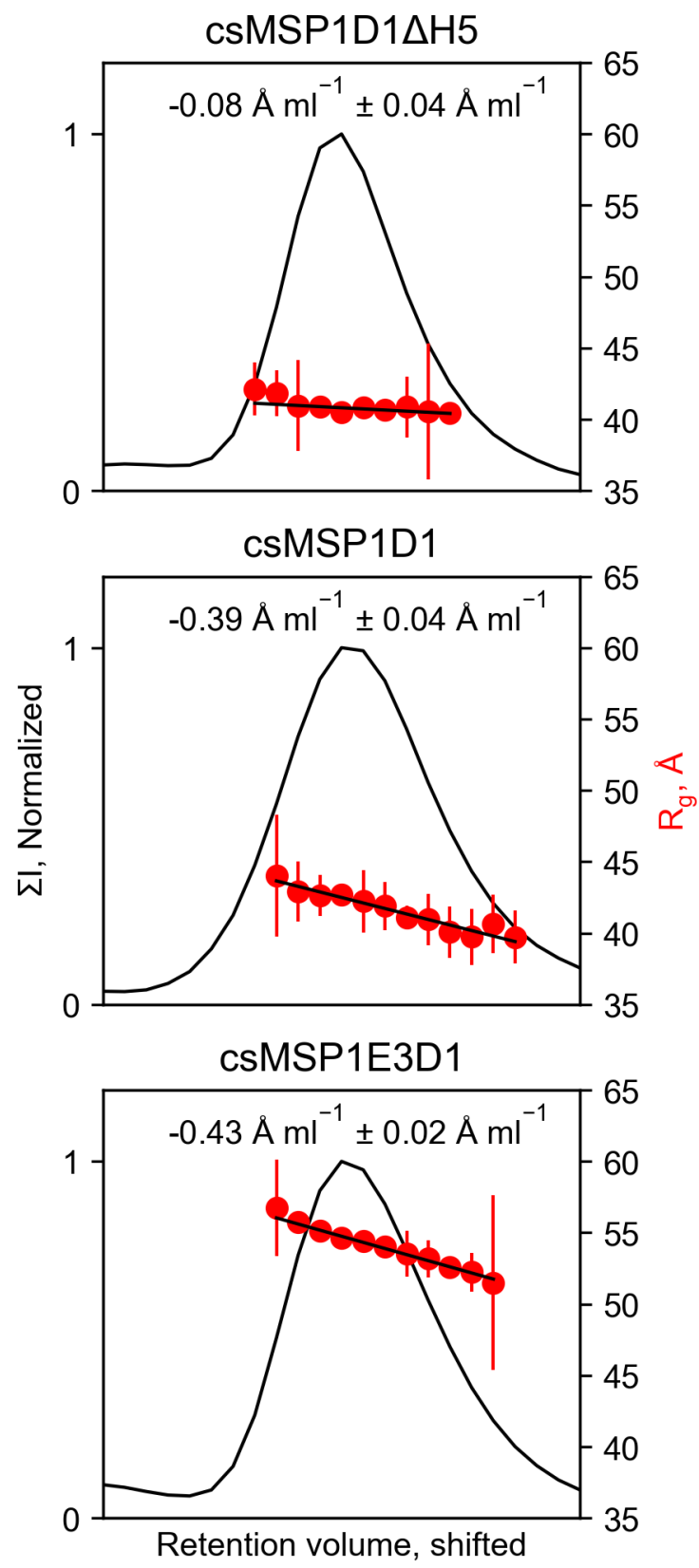

Figure S 2 SEC-SAXS data on cSMSP1D1 $\Delta$ H5, csMSP1D1, and cSMSP1E3D1 nanodiscs with radii of gyration (Rg) calculated for SAXS frames each $0.117 \mathrm{ml}$. The slope of Rg over the peak is given in the plot. 
Figure S 3 - Successive DSC scans
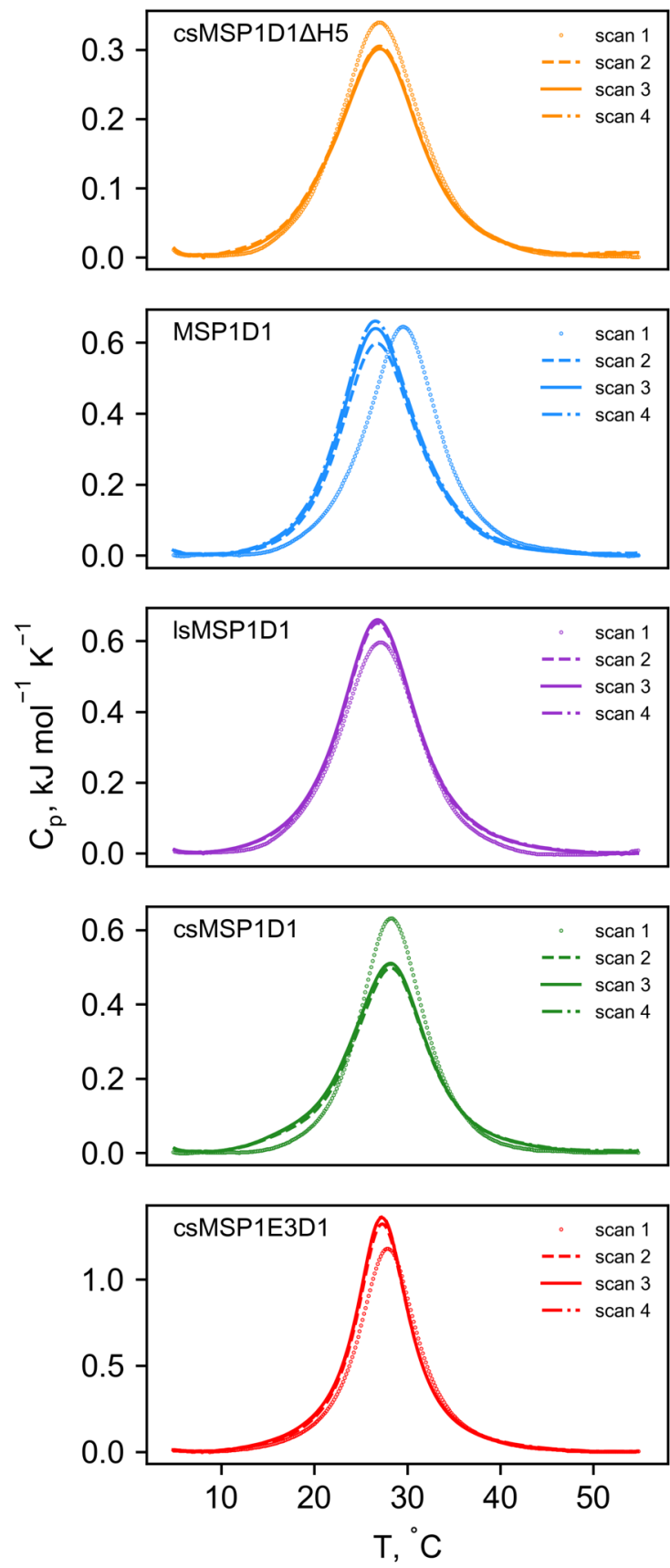

Figure S 3 Successive scans in DSC. Scan 1 is the first up-scan (increasing temperature) after loading the sample, scan 2 is the second up-scan and so forth. The data in the main text corresponds to scan 3. 
Table $\mathbf{S} 4$ - Lipid exchange rates at different concentrations

\begin{tabular}{|c|c|c|}
\hline MSP-DMPC & $C_{D M P C}, \mathrm{mM}$ & $k_{\text {dif }} \cdot 10^{3}, s^{-1}$ \\
\hline \multirow{3}{*}{$\operatorname{csMSP1D1} \Delta \mathrm{H} 5$} & $5.6 \pm 0.4$ & $3.0 \pm 0.2$ \\
\hline & $19.9 \pm 1.4$ & $3.7 \pm 0.1$ \\
\hline & $39.8 \pm 2.7$ & $4.7 \pm 0.0$ \\
\hline \multirow{3}{*}{ MSP1D1 } & $3.9 \pm 0.3$ & $3.7 \pm 0.2$ \\
\hline & $14.1 \pm 0.9$ & $4.8 \pm 0.1$ \\
\hline & $27.5 \pm 1.8$ & $6.1 \pm 0.1$ \\
\hline \multirow{3}{*}{ IsMSP1D1 } & $4.1 \pm 0.3$ & $2.4 \pm 0.1$ \\
\hline & $13.7 \pm 1.0$ & $2.6 \pm 0.0$ \\
\hline & $27.5 \pm 1.9$ & $3.2 \pm 0.0$ \\
\hline \multirow{3}{*}{ csMSP1D1 } & $4.4 \pm 0.2$ & $2.4 \pm 0.1$ \\
\hline & $15.7 \pm 0.8$ & $2.9 \pm 0.0$ \\
\hline & $30.6 \pm 1.7$ & $3.7 \pm 0.0$ \\
\hline \multirow{3}{*}{ csMSP1E3D1 } & $3.4 \pm 0.2$ & $1.6 \pm 0.0$ \\
\hline & $12.2 \pm 0.7$ & $1.9 \pm 0.0$ \\
\hline & $26.7 \pm 1.6$ & $2.0 \pm 0.0$ \\
\hline
\end{tabular}

Table S 4 Lipid monomer exchange rates determined for three different sample concentrations. The rates were measured at $37^{\circ} \mathrm{C} \pm 0.2^{\circ} \mathrm{C}$. 
Figure S 4 - TR-SANS temperature series
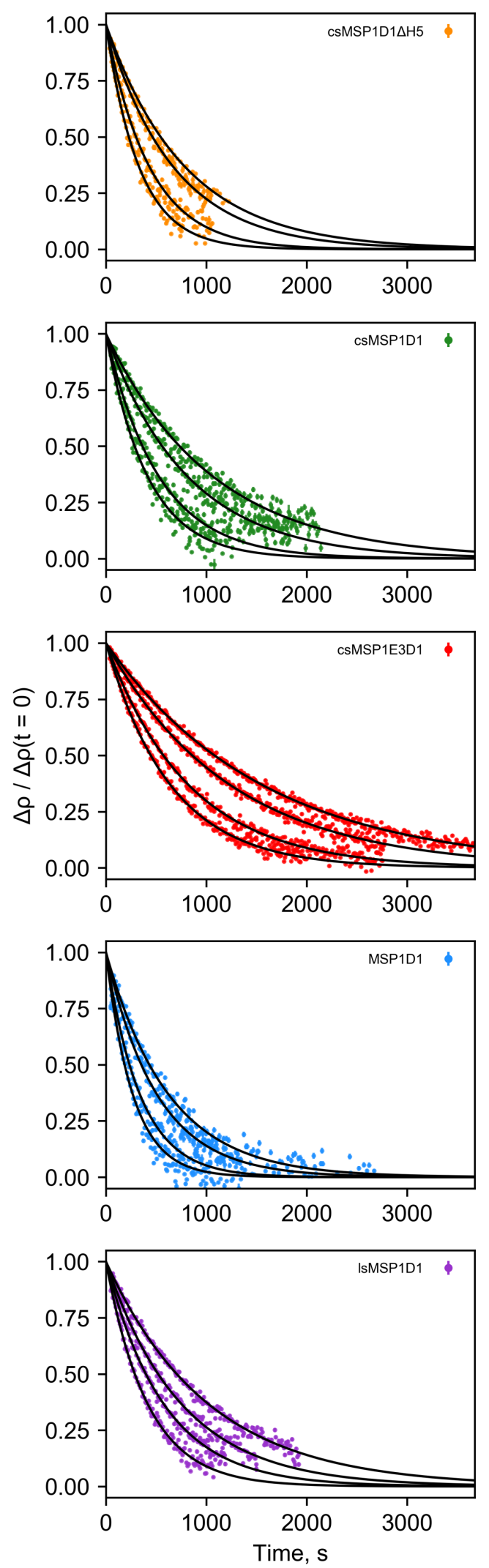

Figure S 4 All collected temperature series of TR-SANS data with single exponential decay fits on top (solid lines). 
Table S 5 - Thermodynamic parameters from lipid exchange

\begin{tabular}{|c|c|c|c|c|c|}
\hline MSP-DMPC & $\begin{array}{c}E_{\mathrm{a}} \\
\mathrm{kJ} \mathrm{mol}^{-1}\end{array}$ & $\begin{array}{c}k_{\mathrm{dif}}{ }^{3{ }^{\circ} \mathrm{C}}\left(\cdot 10^{3}\right) \\
\mathrm{s}^{-1}\end{array}$ & $\begin{array}{l}\Delta \mathrm{G}^{\ddagger}, 37^{\circ} \mathrm{C} \\
\mathrm{kJ} \mathrm{mol}^{-1}\end{array}$ & $\begin{array}{l}\Delta \mathrm{H}^{\ddagger, 37^{\circ} \mathrm{C}} \\
\mathrm{kJ} \mathrm{mol}^{-1}\end{array}$ & $\begin{array}{l}\mathrm{T} \Delta \mathrm{S}^{\ddagger, 37^{\circ} \mathrm{C}} \\
\mathrm{kJ} \mathrm{mol}{ }^{-1}\end{array}$ \\
\hline $\operatorname{csMSP1D1\Delta H5}$ & $106.1 \pm 10.8$ & $6.0 \pm 35.7$ & $89.3 \pm 21.7$ & $103.6 \pm 10.8$ & $14.3 \pm 18.8$ \\
\hline MSP1D1 & $96.3 \pm 8.5$ & $7.4 \pm 34.7$ & $88.7 \pm 17.0$ & $93.7 \pm 8.5$ & $5.0 \pm 14.8$ \\
\hline IsMSP1D1 & $105.2 \pm 6.6$ & $4.8 \pm 17.2$ & $89.9 \pm 13.1$ & $102.6 \pm 6.6$ & $12.8 \pm 11.4$ \\
\hline csMSP1D1 & $109.4 \pm 10.1$ & $4.9 \pm 27.6$ & $89.8 \pm 20.3$ & $106.8 \pm 10.1$ & $17.1 \pm 17.6$ \\
\hline csMSP1E3D1 & $109.4 \pm 2.8$ & $3.2 \pm 4.9$ & $90.9 \pm 5.6$ & $106.9 \pm 2.8$ & $16.0 \pm 4.9$ \\
\hline liposome $^{1}$ & 84.6 & 0.2 & 100.5 & 82.1 & -18.4 \\
\hline$A p o A 1^{2}$ & 98.1 & 4.5 & 91.8 & 95.5 & 3.8 \\
\hline
\end{tabular}

Table S 5 Activation energies, $\mathrm{E}_{\mathrm{a}}$, and lipid monomer exchange rates at $37^{\circ} \mathrm{C}, \mathrm{k}_{\mathrm{dif}}{ }^{3{ }^{\circ} \mathrm{C}}$, extracted from the Arrhenius plot in Figure 5 together with transition state Gibbs free energies, $\Delta \mathrm{G}^{\ddagger}, 37^{\circ} \mathrm{C}$, transition state enthalpies, $\Delta \mathrm{H}^{\ddagger}, 37^{\circ} \mathrm{C}$, and transition state entropies, $\mathrm{T} \Delta \mathrm{S}^{\ddagger}, 37^{\circ} \mathrm{C}$ calculated from the Eyring-Polanyi equation using $\mathrm{E}_{\mathrm{a}}$ and $\mathrm{kdif}^{37^{\circ} \mathrm{C}}$ (see Methods section). 


\section{References}

(1) Nakano, M.; Fukuda, M.; Kudo, T.; Endo, H.; Handa, T. Determination of Interbilayer and Transbilayer Lipid Transfers by Time-Resolved Small-Angle Neutron Scattering. Phys. Rev. Lett. 2007, 98 (23), 30-33.

(2) Nakano, M.; Fukuda, M.; Kudo, T.; Miyazaki, M.; Wada, Y.; Matsuzaki, N.; Endo, H.; Handa, T. Static and Dynamic Properties of Phospholipid Bilayer Nanodiscs. J. Am. Chem. Soc. 2009, 131 (23), 8308-8312. 